\title{
Biopsy of Conjunctiva
}

National Cancer Institute

\section{Source}

National Cancer Institute. Biopsy of Conjunctiva. NCI Thesaurus. Code C51486.

Removal of conjunctival tissue for microscopic examination. 